\title{
Uninformed Consent: Do Medicine Residents Lack the Proper Framework for Code Status Discussions?
}

\author{
Adam F. Binder, MD*, Grace C. Huang, MD², Mary K. Buss, MD, MPH ${ }^{3}$
}

\begin{abstract}
${ }^{1}$ Division of Hematology and Medical Oncology, Icahn School of Medicine at Mount Sinai, New York, New York; ${ }^{2}$ Hospitalist Program, Beth Isreal Deaconess Medical Center, Carl J. Shapiro Institute for Education and Research at Harvard Medical School and Beth Israel Deaconess Medical Center, Boston, Massachusetts; ${ }^{3}$ Ambulatory Palliative Care Services, Division of Hematology/Oncology, Beth Israel Deaconess Medical Center, Boston, Massachusetts.
\end{abstract}

BACKGROUND: Conversations eliciting patient preferences about cardiopulmonary resuscitation (CPR) are among the most common examples of informed consent. However, this is rarely recognized and therefore may not include all key elements of informed consent, namely, details and benefits of the procedure, significant risks involved, likelihood of the outcome, and alternative therapeutic options.

OBJECTIVE: Assess the content of code status discussions as reported by residents to examine whether residents meet requirements of informed consent.

DESIGN: Prospective, observational, single-center survey study.

SETTING: Internal medicine residents at an academic medical center.

INTERVENTION: Medicine residents were surveyed and data were anonymously collected.

MEASUREMENTS: Content of code status discussions and knowledge of CPR outcomes.
RESULTS: Among 100 respondents, $66 \%$ have code status discussions with most patients upon hospital admission. Two main barriers to discussing code status were lack of time (49\%) and lack of rapport (29\%). Only $8 \%$ reported discussing all 5 elements of informed consent. Less than $10 \%$ of the residents correctly answered questions testing knowledge regarding outcomes after cardiac arrest. In logistical regression analyses, residents who included all key elements of informed consent reported more confidence that they provided the information needed for patients to make an informed decision (odds ratio 1.7 [95\% confidence interval: 1.2-2.3]).

CONCLUSIONS: Resident conversations regarding CPR are insufficient in the 5 key elements of informed consent. Framing code status discussions as examples of informed consent may be an effective strategy for educating residents or may improve the quality of these discussions, potentially leading to better patient decisions. Journal of Hospital Medicine 2016;22:111-116. (C) 2015 Society of Hospital Medicine.
Informed consent is one of the ethical, legal, and moral foundations of modern medicine. ${ }^{1}$ Key elements of informed consent include: details of the procedure, benefits of the procedure, significant risks involved, likelihood of the outcome if predictable, and alternative therapeutic options. ${ }^{2}$ Although rarely identified as such, conversations eliciting patient preferences about cardiopulmonary resuscitation (CPR) are among the most common examples of obtaining informed consent. Nevertheless, discussing CPR preference, often called code status discussions, differs from other examples of obtaining informed consent in 2 important ways. First, they occur well in advance of the potential need for $\mathrm{CPR}$, so that the patient is well enough to participate meaningfully in the discussion. Second, because the

*Address for correspondence and reprint requests: Adam F. Binder MD, Division of Hematology and Medical Oncology, Icahn School of Medicine at Mount Sinai, One Gustave L. Levy Place, Box 1079, New York, NY 10029; Telephone: 212-241-8138; Fax: 646-537-8697;

E-mail: adam.binder@mountsinai.org

Additional Supporting Information may be found in the online version of this article.

Received: June 12, 2015; Revised: September 16, 2015; Accepted: September 20, 2015

2015 Society of Hospital Medicine DOI 10.1002/jhm.2497

Published online in Wiley Online Library (Wileyonlinelibrary.com). default assumption is for patients to undergo the intervention (i.e. CPR), the focus of code status discussions is often on "informed refusal," namely a decision about a "do not resuscitate"(DNR) order.

Since the institution of the Patient Self-Determination Act in 1990, hospitals are obliged to educate patients about choices regarding end-of-life care at the time of hospital admission. ${ }^{3}$ In many teaching hospitals, this responsibility falls to the admitting physician, often a trainee, who determines the patient's preferences regarding CPR and documents whether the patient is "full code" or DNR.

Prior studies have raised concerns about the quality of these conversations, highlighting their superficial nature and revealing trainee dissatisfaction with the results. ${ }^{4,5}$ Importantly, studies have shown that patients are capable of assimilating information about CPR when presented accurately and completely, and that such information can dramatically alter their choices. ${ }^{6-8}$ These findings suggest that patients who are adequately educated will make more informed decisions regarding CPR, and that well-informed choices about CPR may differ from poorly informed ones.

Although several studies have questioned the quality of code status discussions, none of these studies frames 


\begin{tabular}{lc}
\hline \multicolumn{2}{l}{ TABLE 1. Respondent Characteristics $(\mathrm{n}=93)$} \\
\hline Characteristic & $\mathrm{N}(\%)$ \\
\hline Sex, male & $50(54)$ \\
PGY level & \\
PGY1 & $35(38)$ \\
PGY2 & $33(35)$ \\
PGY3 & $25(27)$ \\
Exposure to palliative care & \\
Very little & $5(5)$ \\
Occasional & $55(59)$ \\
Frequent & $24(26)$ \\
Completed palliative care elective & $9(10)$ \\
What type of teaching did you have with code status discussions (check all that apply)? & \\
No teaching & $6(6)$ \\
Lectures & $35(38)$ \\
Small group teaching sessions & $57(61)$ \\
Direct observation and feedback & $50(54)$ \\
Exposure to palliative care consultation while rotating on the wards & $54(58)$ \\
Other & $4(4)$ \\
How much has your previous teaching about resuscitative measures influenced your behavior? \\
Not at all & $1(1)$ \\
Not very much & $15(16)$ \\
A little bit & $39(42)$ \\
A lot & $38(41)$ \\
&
\end{tabular}

NOTE: Abbreviations: PGY, postgraduate year.

these interactions as examples of informed consent. Therefore, the purpose of the study was to examine the content of code status discussions as reported by internal medicine residents to determine whether they meet the basic tenets of informed consent, thereby facilitating informed decision making.

\section{METHODS}

In an iterative, collaborative process, authors A.F.B. and M.K.B. (an internal medicine resident at the time of the study and a board-certified palliative care specialist/oncologist with experience in survey development, respectively) developed a survey adapted from previously published surveys. $^{9-11}$ The survey solicited respondent demographics, frequency of code status conversations, content of these discussions, and barriers to discussions. The survey instrument can be viewed in the Supporting Information, Appendix A, in the online version of this article. We used a 5-point frequency scale (almost never-nearly always) for questions regarding: specific aspects of the informed consent related to code status discussions, resident confidence in conducting code status discussions, and barriers to code status discussions. We used a checklist for questions regarding content of code status discussions and patient characteristics influencing code status discussions. Residents provided a numeric percentage answer to 2 knowledge-based questions of postarrest outcomes: (1) likelihood a patient would survive a witnessed pulseless ventricular tachycardia event and (2) likelihood of survival of a pulseless electrical activity event. The survey was revised by a hospitalist with experience in survey design (G.C.H.).
We piloted the survey with 15 residents not part of the subject population and made revisions based on their input.

We sent a link to the online survey over secure email to all 159 internal medicine residents at our urbanbased academic medical center in January 2012. The email described the purpose of the study and stated that participation in the study (or lack thereof) was voluntary, anonymous, and would not have ramifications within the residency program. As part of the recruitment email, we explicitly included the elements of informed consent for the study participants. Not all the questions were mandatory to complete the survey. We sent a reminder e-mail on a weekly basis for a total of 3 times and closed the survey after 1 month. Our goal was a $60 \%(\mathrm{~N}=95)$ response rate.

We tabulated the results by question. For analytic purposes, we aligned the content questions with key elements of informed consent as follows: step-by-step description of the events ("details"), patient-specific likelihood of discharge if resuscitated ("benefits"), complications of resuscitation ("risks"), population-based likelihood of discharge if resuscitated ("likelihood"), and opportunity for changing code status ("alternatives"). For the knowledge-based questions, we deemed the answer correct if it was within $10 \%( \pm 5 \%)$ of published statistics from the 2010 national registry of cardiopulmonary resuscitation. ${ }^{12}$ We stratified the key elements of informed consent and level of confidence by postgraduate year (PGY), comparing PGY1 residents versus PGY2 and PGY3 residents using $\chi^{2}$ tests (or Fisher exact test for observations $\leq 5$ ). We performed a univariate logistic regression analysis examining the relationship between confidence and reported use of informed consent elements in code discussions. The dependent variable of "confidence in sufficient information having been provided for fully informed decision making" was dichotomized as "most of the time" or "nearly always" versus other responses, whereas the independent variable was dichotomized as residents who reported using all 5 informed consent elements versus those who did not. We analyzed data using Stata 12 (StataCorp, College Station, TX).

The institutional review board of the Beth Israel Deaconess reviewed the study protocol and determined that it was exempt from institutional review board review.

\section{RESULTS}

One hundred of 159 (62.3\%) internal medicine residents responded to the survey. Of the respondents $93 \%$ $(\mathrm{N}=93)$ completed the survey. The $7 \%(\mathrm{~N}=7)$ who did not complete the survey omitted the knowledgebased questions and demographics. Approximately half of participants $(54 \%, \mathrm{~N}=50)$ were male. The majority of residents $(85 \%, \mathrm{~N}=79)$ had either occasional or frequent exposure to palliative care, with $10 \%(\mathrm{~N}=9)$ having completed a palliative care rotation (Table 1). 


\begin{tabular}{lc}
\multicolumn{2}{l}{ TABLE 2. Details of Code Status Discussions } \\
\hline \multicolumn{2}{l}{$\mathrm{N}(\%)$} \\
\hline Percentage of inpatients with which you discuss code status, $n=99$ \\
$100 \%$ \\
$81-99 \%$ & $12(12)$ \\
$61-80 \%$ & $53(54)$ \\
$41-60 \%$ & $19(19)$ \\
$21-40 \%$ & $11(11)$ \\
$1-20 \%$ & $3(3)$ \\
Aspects of resuscitative measures routinely discussed, $n=100$ & $1(1)$ \\
Intubation/ventlation & \\
Chest compressions & $100(100)$ \\
Defibrillation & $99(99)$ \\
Surrogate decision maker & $86(86)$ \\
Likelihood of success & $61(61)$ \\
Quality of life & $35(35)$ \\
Vasopressors & $32(32)$ \\
Likelihood of discharge & $13(13)$ \\
Possible role of depression & $10(10)$ \\
Physical states worse than death & $10(10)$ \\
Religious beliefs as a factor & $7(7)$ \\
Makes recommendations for code status, $n=93$ & $6(6)$ \\
Never & \\
Rarely & $19(20)$ \\
Sometimes & $33(35)$ \\
Often & $33(35)$ \\
Nearly always & $7(8)$ \\
& $1(1)$ \\
\hline
\end{tabular}

The vast majority of residents $(96 \%, \mathrm{~N}=95)$ discussed code status with more than $40 \%$ of patients they admitted to the hospital (Table 2). Two-thirds (66\%, N $=65)$ of all residents had the conversation with at least 4 out of $5(81 \%-99 \%$ and $100 \%)$ patients they admitted to the hospital. Only $1 \%(\mathrm{~N}=1)$ of residents who responded to the survey reported conducting code status discussions with $20 \%$ or fewer of the patients they admitted to the hospital.

Most residents $(66 \%, \mathrm{~N}=66)$ identified the healthcare proxy or surrogate decision maker most of the time or nearly always. In addition, most residents $(62 \%, N=62)$ reminded patients that they could reverse their code status at any time. Almost half included a description of step-by-step events during resuscitation $(45 \%, \mathrm{~N}=45)$ or factored in patient's comorbidities $(43 \%, \mathrm{~N}=43$ ) when discussing resuscitation at least most of the time. Few residents described complications $(31 \%, \mathrm{~N}=31)$ or outcomes $(17 \%, N=17)$ of cardiopulmonary arrests to patients "most of the time" or "nearly always." Most residents did not explore factors such as quality of life, role of depression or physical states worse than death, factors that could potentially affect patient decision making (Table 2). Few $(9 \%, \mathrm{~N}=8)$ internal medicine residents ("often" or "nearly always") offered their opinion regarding a patient's code status.

Many factors influenced residents' decisions to have a code status conversation. At least $85 \%(\mathrm{~N}=86)$ of residents reported that older age, particular admitting diagnoses, and multiple comorbidities made them more likely to have a code status discussion (see Sup- porting Table 1 in the online version of this article). Patient race/ethnicity did not influence this decision, with only 1 respondent reporting this factor as relevant.

Residents identified lack of time $(49 \%, \mathrm{~N}=49$ responding "often" or "nearly always") as the most frequent barrier to having a code status discussion, followed by lack of rapport $(29 \%, \mathrm{~N}=29)$. Lack of experience $(6 \%, \mathrm{~N}=6)$, lack of information about the patient's clinical status $(11 \%, \mathrm{~N}=11)$, and lack of knowledge about outcomes $(13 \%, \mathrm{~N}=13)$ did not represent frequent barriers for residents.

Fifty-five percent $(\mathrm{N}=53)$ of residents "often" or "nearly always" felt confident that they provided enough information for patients to make fully informed decisions about code status, and this did not differ by PGY status (PGY1 vs PGY2/3, $P=0.80, \chi^{2}$ test). However, only $8 \%(\mathrm{~N}=8)$ of residents "most of the time" or "nearly always" addressed all 5 key elements of informed consent in reporting the content of their code status discussions. When stratified by training year, PGY2/3 residents were significantly more likely than PGY1 residents to factor in a patient's comorbidities when discussing resuscitation and were also significantly more likely to relay the likelihood of hospital discharge. They were not significantly more likely to discuss other key elements of informed consent (Table 3).

Our subanalysis showed that residents reporting all 5 key elements of informed consent were associated with higher levels of confidence that they had provided enough information to patients for them to make an informed decision (odds ratio of 1.7, 95\% confidence interval 1.2-2.3).

For the first knowledge-based question about witnessed pulseless ventricular tachycardia, according to the 2010 registry, ${ }^{12} 64 \%$ survived the event (range of responses $1 \%-90 \%)$. Six out of $92(7 \%)$ respondents were within $5 \%$ of the correct answer. For the second question about survival after unwitnessed pulseless electrical activity, $41.5 \%$ survived the event according to the registry (range of responses $1 \%-50 \%$ ). Three out of $92(3 \%)$ respondents gave estimates within $\pm 5 \%$ of the correct answer. Figures 1 and 2 display the ranges of responses from residents.

\section{DISCUSSION}

We found that although our internal medicine residents frequently have code status discussions with their patients, very few routinely report addressing all 5 key elements of informed consent. Furthermore, residents lack accurate knowledge about the outcomes of CPR, with most tending to underestimate the "benefit" expected of resuscitation. These deficiencies raise serious concerns about whether patients are receiving all the information essential to making fully informed decisions about their preferences for resuscitation. 
TABLE 3. Key Elements of Code Status Discussions

\begin{tabular}{|c|c|c|c|c|c|}
\hline Elements of Code Status Discussion (Most of the Time or Nearly Always), $n=100$ & Elements & Total, N (\%) & PGY1, N (\%) & PGY2/3, N (\%) & $P$ Value \\
\hline Identify the patient's HCP or surrogate & & $66(66)$ & N/A & N/A & N/A \\
\hline Describe the step-by-step events that occur during resuscitative measures & Details & $45(45)$ & $14(40)$ & $28(33)$ & 0.437 \\
\hline Describe the complications associated with resuscitative measures & Risks & $31(31)$ & $8(23)$ & $19(33)$ & 0.308 \\
\hline Describe the likelihood the patient will be discharged from the hospital if resuscitated & Likelihood & $17(17)$ & $2(6)$ & $14(24)$ & 0.025 \\
\hline $\begin{array}{l}\text { Factor in the patient's comorbidities when discussing the likelihood } \\
\text { of discharge from the hospital if resuscitated }\end{array}$ & Benefits & $43(43)$ & $8(23)$ & $33(57)$ & 0.002 \\
\hline Tell the patient that decisions regarding code status can be changed at any time & Alternatives & $62(62)$ & $18(51)$ & $38(66)$ & 0.179 \\
\hline
\end{tabular}

NOTE: Abbreviations: HCP, healthcare proxy; N/A, not applicable; PGY, postgraduate year.

The data demonstrate that the residents are routinely discussing code status and regularly discussing some aspects of the procedure itself, such as chest compressions, intubation, or defibrillation; the actual step-by-step events of CPR are being described less than half the time. It seems that residents mentally list the possible procedures that may occur in a code without a context for how one intervention would lead to another. Placing CPR into context is important, because studies have shown that more comprehensive discussions or the use of visual aids/videos that depict CPR in more detail improves patients' understanding of CPR and changes their decision about CPR, making them more likely to forego the procedure. ${ }^{7,8}$

Residents report that they are more likely to have a code status discussion with patient's with multiple comorbidities, suggesting that they take into account "information about the patient's clinical condition" when deciding with which patients to address code status. They also recognize which patients are at increased risk for an in hospital cardiopulmonary arrest. Additionally, nearly half of residents "factor in patient's comorbidities when discussing likelihood of discharge from the hospital," suggesting that they recognize that comorbidities can alter the outcome of CPR. Importantly, however, very few residents "describe the likelihood the patient will be discharged from the hospital if resuscitated." Thus, residents in our sample have some
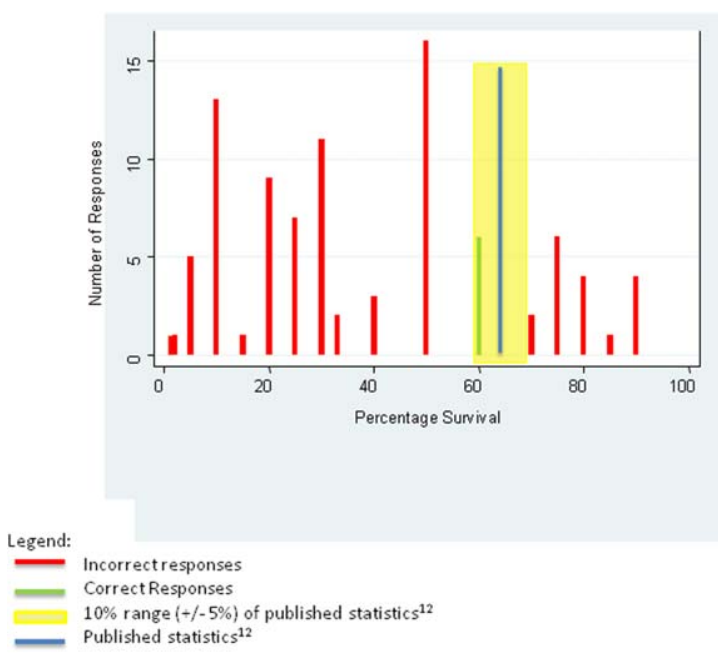

FIG. 1. Responses to the likelihood a patient would survive an in-hospital, witnessed, pulseless, ventricular tachycardia event. insight into the impact of comorbidities on outcomes of CPR, but fail to provide their patients with any information about the outcome of CPR.

One reason residents may not discuss outcomes of $\mathrm{CPR}$ is because they do not know the data regarding outcomes. Although few residents reported that lack of knowledge of the risks and outcomes of CPR was a barrier, very few respondents answered the knowledge questions appropriately. Given how few residents gave an accurate estimate of CPR outcomes and simultaneously reported confidence in their code status discussions suggests that many residents fail to recognize their knowledge deficits. This finding corroborates other studies showing that residents "don't know what they don't know" 10 and may reflect their lack of education on CPR outcomes. Alternatively, some residents who underestimated the outcomes in the examples provided may have done so because, in their experience caring for patients with multiple comorbidites, the outcomes of CPR are in fact poorer than those in the cases described. Outcomes of CPR at our institution might differ from those quoted in the registry. However, given the prevalence of inaccuracy, both for under- and overestimation, it seems likely that a true knowledge deficit on the part of the residents still accounts for much of the error and should

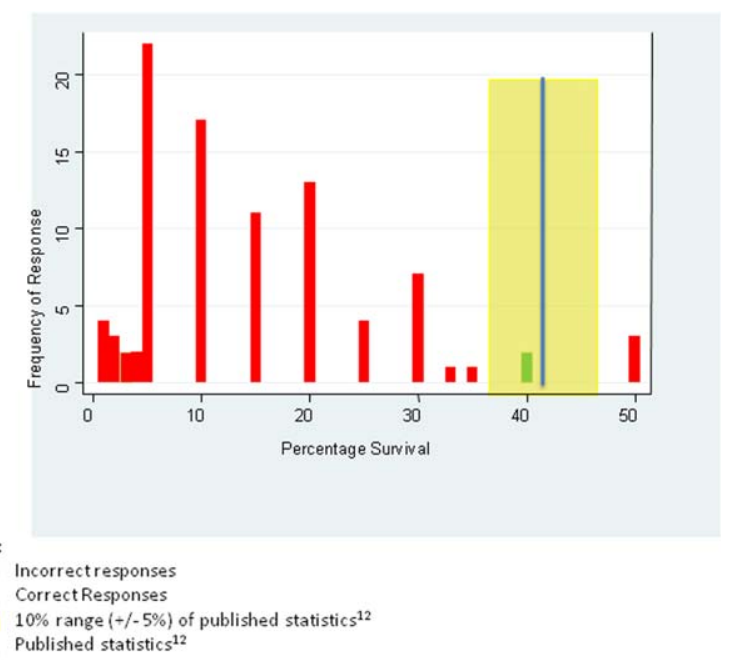

FIG. 2. Responses to the likelihood a patient would survive an in-hospital, pulseless, electrical activity event. 
be a target for education. Understanding CPR outcomes is vital for informed decision making, and studies have shown that when patients have more information, it can substantially affect a patient's decision regarding resuscitation., ${ }^{73}$

Residents are infrequently exploring key determinants that affect a patient's decision-making process. Only one-third of residents report discussing qualityof-life issues with patients during code status discussions. Understanding an individual patient's values and goals and how he or she describes a good quality of life can help guide the discussion and potential recommendations. For example, some patients may feel it is important to be alive regardless of the physical state, whereas others may feel that if there is not a chance to be independent in their activities of daily living, then they would not want to be resuscitated. By exploring patient's perceptions of what quality of life and physical states worse than death means, residents can better understand and assist in the decisionmaking process of their patients.

Our data show that few residents offer a recommendation regarding code status. Thus, residents expect patients to make their own decision with the data provided. At the same time, many residents focus on the details of the procedural components of CPR with little mention of anticipated outcomes or inquiries into key determinants discussed above. Additionally, based on their response to the knowledge-based questions, residents' estimates of survival, if offered, would be inaccurate. Thus, code status conversations by residents leave patients to make uninformed choices to consent to or refuse resuscitative measures.

When stratified by training year, PGY $2 / 3$ residents were significantly more likely than PGY1 residents to discuss likelihood of discharge from the hospital as well as factor in patients' comorbidities when discussing outcomes. Although there is a statistically significant improvement between PGY2/3 residents as compared to PGY1 residents, the numbers still show that most PGY2/3 residents and almost all PGY1 residents do not discuss the likelihood of discharge if resuscitated during code status discussions. In addition, there is no difference reported in other key areas of informed consent. Thus, though there is some improvement as housestaff advance in their training, PGY2 and PGY3 residents still do not discuss all 5 key elements of informed consent significantly more than PGY1 residents.

Our findings suggest an opportunity for additional education regarding how to address code status for internal medicine housestaff. Over half of the respondents reported small group teaching sessions, direct observation and feedback, and exposure to palliative care consultation during their clinical rotations; yet, very few of them included all the key elements of informed consent in their discussions. To address this, our institution is developing additional educational initiatives, including a faculty development program for teaching communication skills, using direct observation and feedback. The orientation didactic lecture series for housestaff now includes a lecture on CPR that highlights the data on outcomes and the importance of putting the step-by-step procedures of CPR into the context of potential benefits, such as survival to hospital discharge. The curriculum also includes a module on advance care planning for junior and senior residents during their ambulatory block, using simulation and feedback as part of the teaching methods.

There are limitations to this study. Studies based on surveys are subject to recall and selection bias, and we lack objective assessment of actual code status discussions. Furthermore, the nature of the study may lead to an overestimation of the quality of the code status discussions due to social acceptability bias; yet, our data clearly show that the key elements of informed consent are not included during these conversations. Another limitation is that our subjects were residents at a single institution, and our clinical practice may differ from other academic settings in the teaching environment and culture; yet, our findings mirror similar work done in other locations. ${ }^{10,14}$

In conclusion, our results demonstrate that residents fail to meet standards of informed consent when discussing code status in that they do not provide sufficient information for patients to make an informed decision regarding resuscitation. Residents would benefit from education aimed at improving their knowledge of CPR outcomes as well as training on how to conduct these conversations effectively. Framing code status discussions as an example of an informed consent may help residents recognize the need for the key elements to be included in these conversations. In addition, training should focus on how to conduct these conversations in an efficient yet effective manner. This will require clear simple language, good communication skills, and training with observation and feedback by specialists trained in this field.

Disclosures: This work was presented at the Society of General Internal Medicine New England Regional Meeting, March 8, 2013, Yale Medical Center, New Haven, Connecticut. The authors report no conflicts of interest.

\section{References}

1. Paterick TJ, Carson GV, Allen MC, Paterick TE, Medical informed consent: general considerations for physicians. Mayo Clin Proc. 2008; 83(3):313-319.

2. Beth Israel Deaconess Medical Center. Policy \#PR-02 45 CFR 46.116\&21 CFR 50.25.

3. Koch KA. Patient self-determination act. J Fla Med Assoc. 1992;79(4): 240-243.

4. Deep KS, Green SF, Griffith CH, Wilson JF. Medical residents' perspectives on discussions of advanced directives: can prior experience affect how they approach patients? J Palliat Med. 2007;10(3):712-720.

5. Anderson WG, Chase R, Pantilat SZ, Tulsky JA, Auerbach AD. Code status discussions between attending hospitalist physicians and medical patients at hospital admission. J Gen Intern Med. 2010;26(4):359-366.

6. Murphy DJ, Burrows D, Santilli S. The influence of the probability of survival on patient's preferences regarding cardiopulmonary resuscitation. N Engl J Med. 1994;330:545-549.

7. Volandes AE, Mitchell SL, Gillick MR, Chang Y, Paasche-Orlow MK. Using video images to improve the accuracy of surrogate decision- 
making: a randomized controlled trial. J Am Med Dir Assoc. 2009; 10(8):575-580.

8. El-Jawahri A, Podgurski LM, Eichler AF, et al. Use of video to facilitate end-of-life discussions with patients with cancer: a randomized controlled trial. J Clin Oncol. 2010;28(2):305-310.

9. Smith AK, Ries AP, Zhang B, Tulsky JA, Prigerson HG, Block SD. Resident Approaches to Advance Care Planning on the Day of Hospital Admission. Arch Intern Med. 2006;166:15971602.

10. Buss MK, Alexander GC, Switzer GE, Arnold RM. Assessing competence of residents to discuss end-of-life issues. J Palliat Med. 2005; 8(2):363-371.
11. Kaldjian LC, Erekson ZD, Haberle TH, et al. Code status discussions and goals of care among hospitalised adults. J Med Ethics. 2009;35: 338-342.

12. Larkin GL, Copes WS, Nathanson BH, Kaye W. Pre-resuscitation factors associated with mortality in 49,130 cases of in-hospital cardiac arrest: a report from the National Registry for Cardiopulmonary Resuscitation. Resuscitation. 2010;81:302-311.

13. Schonwetter RS, Walker RM, Kramer DR, Robinson BE. Resuscitation decision making in the elderly: the value of outcome data. J Gen Intern Med. 1993;8:295-300.

14. Tulsky JA, Chesney MA, Lo B. How do medical residents discuss resuscitation with patients? J Gen Intern Med. 1995;10:436-442. 